\title{
Redefining Quality in Endoscopic Sedation
}

\author{
Lawrence B. Cohen
}

Published online: 29 July 2010

(C) Springer Science+Business Media, LLC 2010

The popularity of anesthesia-assisted sedation is attributable to two closely interwoven elements of this practice: namely the drug propofol, and the presence of an additional individual to assist with drug administration and patient monitoring. Propofol, a short-acting sedative hypnotic agent, has a rapid onset of action and quick offset. Patients awaken rapidly from propofol with a feeling of clearheaded recovery. Propofol is capable of producing deep sedation and general anesthesia, distinguishing it from the benzodiazepines that exhibit a ceiling effect with regard to the depth of sedation. Consequently, propofol's attraction as an agent for procedural sedation is a direct result of its pharmacokinetic and pharmacodynamic properties.

Notwithstanding propofol's desirable properties, we must also acknowledge the valuable contribution of the anesthesia provider. This individual improves practice efficiency by assisting with the pre-procedural assessment, preparation of the patient including placement of the intravenous catheter and monitoring electrodes, intraprocedural monitoring and administration of drugs, and recovery room care. As a result, operating efficiency is improved and staffing costs may be reduced. These benefits come at a price, however. The average cost of anesthesiaassisted sedation during an endoscopic procedure is roughly $\$ 400$ per case [1]. This cost is ultimately passed on to patients in the form of higher health insurance

L. B. Cohen $(\square)$

The Mount Sinai School of Medicine, New York, NY 10029,

USA

e-mail: Lawrence.Cohen@nyga.md

L. B. Cohen

311 East 79th Street, New York, NY 10075, USA premiums, and this must be considered when examining the overall effectiveness of anesthesia services.

In this issue of Digestive Disease and Sciences, Nayar and colleagues report the results of their retrospective analysis comparing the outcomes of 1,000 endoscopic ultrasound (EUS) procedures performed with anesthesiaassisted propofol sedation and 1,000 EUS procedures performed under conventional sedation using a benzodiazepine and an opioid [2]. The two groups were noncontemporaneous, with the conventional sedation and anesthesia care cohorts enrolled sequentially during two consecutive 2-year intervals, beginning on January 1, 1999. As anticipated, sedation induction time and recovery time were significantly shorter in the group receiving propofol (3.1 and $35 \mathrm{~min}$ ) compared to those receiving standard sedation (5.8 and $70 \mathrm{~min}$ ). Unexpectedly, intra-procedural time was significantly reduced in the propofol group (28 $\mathrm{min}$ ) compared to patients sedated with a benzodiazepine and an opioid (61 min). Patient satisfaction was higher with propofol (9.5) compared to traditional sedation (8.3) using a ten-point visual analogue scale $(10=$ best $)$, although both patient cohorts reported good levels of satisfaction.

Notably, the rate of sedation-related complications was numerically higher in the anesthesia group compared to patients receiving standard sedation, although the difference was not statistically significant. A total of six complications were observed in the anesthesia care group, including perforation [1], aspiration [1], apnea requiring endotracheal intubation [3], and hypotension [1]. In contrast, only one complication (prolonged somnolence) was recorded in the moderate sedation group. The treatment groups were similar with respect to age, gender, BMI, study indication, and ASA physical classification, although numerically, there were fewer ASA 1 and $2 \mathrm{~s}$, and more 
ASA 3 and $4 \mathrm{~s}$, in the propofol group compared to the standard sedation group.

There are few randomized controlled trials comparing the efficiency, safety, and patient satisfaction between propofol and traditional sedation. A meta-analysis of three studies (two colonoscopy and one colonoscopy or EGD), all involving propofol administration by an endoscopist, detected no significant difference in the incidence of adverse events, physician satisfaction, or patient discomfort or pain between the two sedation groups [3]. Patientreported satisfaction was greater, and sedation induction and recovery times were shorter, with propofol compared to midazolam and an opioid. Similar findings were observed in a small, prospective study that compared sedation outcomes during EUS in patients randomized to nurse-administered propofol or midazolam and meperidine. The use of propofol was associated with faster induction and recovery of sedation, higher postprocedure patient satisfaction, and quicker return to baseline function. Procedure times and the frequency of adverse events were similar in both groups [4]. As noted previously, propofolbased sedation improved operational efficiency and patient satisfaction compared to traditional methods of sedation.

We know less about the comparative effects of various sedation methods on the quality of endoscopic examinations. In one study, expert observational analysis of videotaped procedures was used to compare the quality of EGDs performed under midazolam versus propofol [5]. Examinations were assessed by three blinded endoscopists using a standardized score sheet. Nearly all parameters of the endoscopic examination were superior with propofol compared to midazolam. In a similar study involving EUS, investigators used observational analysis and a validated scale in order to compare sedation quality between anesthesia-assisted propofol and benzodiazepine/opioid combination [4]. Once again, the quality of sedation was adjudged to be superior with propofol with fewer episodes of under- and over-sedation, quicker sedation induction and recovery times, and higher ratings for patient satisfaction. More work in this area is needed in order to define the true cost-effectiveness of anesthesia-assisted propofol sedation.

It is unclear why intra-procedural times in this study were more than twice as long with traditional sedation compared to anesthesia-assisted sedation. There are several possible explanations for this. It is conceivable that improvements in technology during the 4-year period of study may have simplified the technical performance of EUS, reducing the time necessary to perform a complete examination. Perhaps differences in case mix between the two patient cohorts could have also accounted for the variation in procedure times. Or maybe there were differences in the proportion of cases being performed by trainees as compared to experienced attending. Regardless of the cause(s), the results of other EUS studies indicate that the method of sedation is unlikely to account for the difference in procedure length observed by Nayar.

This study recorded more cardiopulmonary events in the group receiving monitored anesthesia care than those receiving traditional sedation. While the difference was not statistically significant, it provides an important signal. The literature provides little data on the rate of complications associated with anesthesia-assisted sedation, although it is generally believed that the incidence is greater in monitored anesthesia care than with moderate sedation or general anesthesia. A recent prospective study evaluating sedation-related complications in patients undergoing advanced endoscopic procedures with anesthesia-assisted sedation reported 154 airway maneuvers in 115 patients (14.4\%) including modified face mask in 29 (3.6\%) [6]. Observations from the American Society of Anesthesiology Closed Claims database indicate that anesthesia care at remote locations, such as the gastrointestinal endoscopy suite, poses a significant risk for the patient related to oversedation and ventilatory failure [7]. Importantly, the severity of these injuries was greater than those occurring within the operating room, and more than half of all sedation-related complications resulted in death. Thus, we must define clinically important adverse events related to sedation and compare rates of occurrence for various methods of sedation.

Perhaps it is time to redefine quality benchmarks for sedation in gastrointestinal endoscopy. Naturally, patient safety must be our number one priority. If anesthesiaassisted sedation is to become an integral element of endoscopic practice, we must establish that it improves the safety of sedation compared to traditional methods. Currently, there are no reliable data addressing this issue. We must also evaluate how various sedation strategies impact the quality of endoscopy. This will require that we establish evidence-based measures designed to assess the quality of an endoscopic examination. This will necessarily be easier for some procedures than for others. For example, adenoma detection rate has recently been validated as an indicator for the quality of colonoscopy. Other examples could include cannulation rates during ERCP and successful homeostasis of bleeding. While practice efficiency and patient satisfaction are useful measures of sedation quality, they are insufficient without evidence attesting to safety and effectiveness.

In conclusion, we need to better understand the impact of anesthesia-assisted sedation on the risk of sedation-related adverse events and the quality of endoscopy. Randomized trials that compare traditional sedation with anesthesiaassisted sedation for patient safety and endoscopic outcomes are warranted. The study by Nayar and colleagues expands our understanding about anesthesia-assisted sedation during 
EUS, but it leaves several key questions about its efficacy and cost-effectiveness unanswered.

Conflict of interest The authors report no relevant conflicts of interest.

\section{References}

1. Aisenberg J, Brill JV, Ladabaum U, Cohen LB. Sedation for gastrointestinal endoscopy: new practices, new economics. Am J Gastroenterol. 2005;100(5):996-1000.

2. Nayar DS, Guthrie WG, Goodman A, Lee Y, Feuerman M, Scheinberg L et al. Comparison of propofol deep sedation versus moderate sedation during endosonography. Dig Dis Sci. 2010. doi: 10.1007/s10620-010-1308-0.
3. McQuaid KR, Laine L. A systematic review and meta-analysis of randomized, controlled trials of moderate sedation for routine endoscopic procedures. Gastrointest Endosc. 2008;67:910-923.

4. Trummel JM, Surgenor SD, Cravero JP, Gordon SR, Blike GT. Comparison of differing sedation practice for upper endoscopic ultrasound using expert observational analysis of the procedural sedation. J Patient Saf. 2009;5:153-159.

5. Meining A, Semmler V, Kassem AM, et al. The effect of sedation of the quality of upper gastrointestinal endoscopy: an investigatorblinded, randomized study comparing propofol with midazolam. Endoscopy. 2007;39:345-349.

6. Cote GA, Hovis RM, Ansstas MA, et al. Incidence of sedationrelated complications with propofol during advanced endoscopic procedures. Clin Gastroenterol Hepatol. 2010;8:137-142.

7. Metzner J, Posner KL, Domino KB. The risk and safety of anesthesia at remote locations: the US closed claims analysis. Curr Opin Anesthesiol. 2009;22:502-508. 